\title{
Fuzzy analytical hierarchy process using preference ratio: A case study for selecting management short course in a business school
}

\author{
${ }^{c}$ Industrial Management Institute, Tehran, Iran

\begin{tabular}{l}
\hline A R T I C L E I N F O \\
\hline Article history: \\
Received 1 January 2010 \\
Received in revised form \\
10 May 2010 \\
Accepted 18 May 2010 \\
Available online 18 May 2010 \\
\hline Keywords: \\
FAHP \\
Preference ratio \\
MADM \\
Fuzzy numbers \\
Consistency ratio \\
AHP
\end{tabular}

Mohammad Modarres $^{\mathrm{a}}$, Soheil Sadi-Nezhad ${ }^{\mathrm{b}^{*}}$ and Farzane Arabi ${ }^{\mathrm{c}}$

${ }^{a}$ Department of Industrial Engineering,Sharif University of Technology, Tehran, Iran

${ }^{b}$ Department of Engineering,Islamic Azad University, Science \& Research Branch, Tehran, Iran

A B S T R A C T

\begin{abstract}
Analytic Hierarchy Process (AHP) is one of the most popular approaches in the area of multiple attribute decision making (MADM). However, it is not practical any more if input information are fuzzy. In this paper, we propose a new method for fuzzy AHP which is especially useful to make decisions for multiple attribute problems. The method is developed by applying preference ratio concept which makes it practical since it assigns crisp weights and crisp scores to different alternatives. Two algorithms are proposed in this paper: The first one defines crisp and normalized weight by pairwise comparison with fuzzy data while the second one calculates fuzzy consistency ratio. The proposed method is applied to prioritize different short courses in a management school.
\end{abstract}

(c) 2010 Growing Science Ltd. All rights reserved.

\section{Introduction}

The Analytic Hierarchical Process (AHP) method proposed by Saaty (1977) uses pair-wise comparisons to compute the performance score of alternatives with respect to each attribute. The pair-wise comparison matrix is also applied for each attribute to compute their appropriate weights. In Saaty's approach, the pairwise comparison ratios are real numbers. Therefore the resulting weights and performance scores are also crisp and real numbers. According to Saaty and Vargas (1994), uncertainty in judgments can be expressed either as a point estimate with a probability distribution function or as an interval estimate without probability distribution. Traditional AHP method does not directly use fuzzy numbers or membership functions to express fuzzy information. Instead, it uses the estimation of an underlying ratio scale together with consistency measure in order to consider fuzziness of a multiple attribute decision-making (MADM) problem. Laurhoven and Pedrycz (1985) proposed a method of fuzzy judgment using triangular fuzzy numbers (TFN). They applied arithmetic operations for TFNs and logarithmic least square method to calculate fuzzy utilities. Chen and Hwang (1992) listed some disadvantages for this approach such as:

1. There is not always a feasible solution to the proposed equations.

2. Even if there is a feasible solution, the results may not be a TFN.

\footnotetext{
* Corresponding author. Tel:(98)(21)22010690./fax: (98)(21)22043001

E-mail addresses: sadinejad@hotmail.com (S. Sadinezhad),

C 2010 Growing Science Ltd. All rights reserved.

doi: $10.5267 /$ j.ijiec.2010.02.006
} 
3. The burden of the computation is relatively high.

In literature, there are several approaches to deal with this type of problem. Buckley (1985) used a method on the basis of geometric mean to derive fuzzy weights and performance score. This method ends up with fuzzy numbers. Therefore, we need to compare them by a fuzzy ranking method. Cheng (1996) proposed the principle for comparison between the elements of the fuzzy numbers and used degree of possibility as a concept in his work. Chang (1996) developed extent analysis (EA) method where the value of each criterion was calculated by EA method. They also used a specific probability concept to compare two fuzzy numbers. The most disadvantage of this method is the ignorance of consistency in pair-wise comparison matrices. Sallo was the first who pointed it out the consistency condition and Chen and Chong (Leng \& Cao 2000) defined them based on crisp weights. Zhu et al. (1999) also proved the basic theory of the TFN and improved the formulation of comparing the TFNs. Yeh et al. (2000) developed an eleven-step method and defined the degree of optimality for each alternative. Later, they used Hamming distance between ideal points. Buckly et al. (2001) presented a new method of finding the fuzzy weights in fuzzy hierarchical analysis (FHA) which is the direct fuzzification of the original method used by Saaty in the AHP. They also recommended fuzzifying the geometric mean procedure to obtain weights in FHA. The calculation of fuzzy weights is quite complicated so they used EA to search for Min and Max in their research. Mikhailov (2004) studied the problem of priority elicitation in the AHP and proposed a new approach deriving crisp priorities from interval pairwise comparison judgments. They used a fuzzy mathematical programming approach to find an optimal crisp priority vector and suggested a consistency index which measures the level of inconsistency of interval judgments. Saaty (2006) came up with a new idea about ranking from comparisons and from rating in the AHP and analytical network process. They deal with rank preservation and reversal as an important subject in MCDM.

Today, fuzzy AHP has a special place in fuzzy MADM problems for real cases such as: technology transfer strategy selection (Chang and Chen 1994); evaluation of IT Services (Mikhailov et al. 2004); assessment in the managerial talent in Taiwan's semiconductor industry (Huang \& Wu 2005); evaluating attack helicopter (Ching et al. 1999); performance evaluation of bus companies (Yeh et al. 2000); Evaluation and pre-allocation of operators with multiple skills ( Şen \& Çinar 2010); determine the relative weights of evaluation criteria to rank the alternatives (Torfi et al. 2010); Evaluation of factors influencing knowledge sharing (Lin et al. 2009); government-sponsored R\&D project selection (Huang et al. 2008).

Modarres and Sadi-Nezhad (2001) proposed a technique for normalizing fuzzy information by using preference ratio, which is applied in this paper for AHP problems with fuzzy information. In the next section, the concept of preference ratio is introduced as well as the definition of equivalence by preference ratio. Then, in section 3 we propose fuzzy AHP method by applying preference ratio approach and a numerical example is presented to demonstrate the implementation of the proposed method. An algorithm for calculating fuzzy consistency ratio and an example is also presented in section 4. At the end, we apply this method for selecting management short course as a training program in a business school for offering to CEOs.

\section{Preference Ratio}

Our proposed approach is developed based on the concept of preference ratio as well as ranking method introduced by Modarres and Sadi-Nezhad (2001) where we evaluate fuzzy numbers "point by point" and rank them at each point. Then, the overall preference over all points is calculated and the preference is relative rather than absolute. Suppose the objective is to rank $I$ fuzzy numbers. Let $N_{i}$ be the ith one defined over a real domain $S_{i} \subset R$, and is identified by a membership function $\left(\mu_{N_{i}}(x), x \in S_{i}\right)$, with $\mu_{N_{i}} \in[0,1]$. Let $S_{i}$ be the support of $N i$, or more precisely $S_{i}=\left\{x, \mu_{N_{i}}(x)>0\right\}$, and $\Omega=U_{i=1}^{1} S_{i}$, then $\Omega$ is the union of the support of all fuzzy numbers. In other word, fuzzy numbers are ranked over $\Omega$. (To rank fuzzy numbers, we make a simple assumption that their spans 
are not disjoint, because in that case the ranking is clear). A fuzzy number is evaluated by a function called Preference Function. At each point $\alpha \in \Omega$, this function is defined as follows,

$G(\alpha)=\frac{\int_{\alpha}^{U} \mu(x) d x}{\int_{L}^{U} \mu(x) d x}$

where $\mu(x)$ is the membership function of the fuzzy number, $L=\min \{x: x \in \Omega\}$ and $U=\max \{x: x \in \Omega$ \}. This function has the same definition as $1-F(\alpha)$ in probability theory, where $F(\alpha)=P[X \leq \alpha]$ is the distribution function. Let $\alpha \in \Omega$, let $p(\alpha)=i$ denote the ith fuzzy number, which is the most preferred one. Therefore,

$p(\alpha)=i, \quad$ if $\quad G_{i}(\alpha)=\max \left\{G_{j}(\alpha), j \in I\right\}$

where, $G_{j}(\alpha)$ is the preference function of the $j$ th fuzzy number. Let, $\Omega_{i}$ be the set of points in which the $i$ th number is ranked number one. Then,

$\Omega_{i}=\{\alpha \in \Omega, p(\alpha)=i\}$.

Definition 1: For the ith fuzzy number, $R(i)$, the Preference Ratio is, by definition, the percentage of $\Omega$ that the ith fuzzy number is the most preferred one. Then,

$R(i)=\frac{\left|\Omega_{i}\right|}{|\Omega|}$,

where $\left|\Omega_{i}\right|$ and $|\Omega|$ are the lengths of the real set $\Omega_{i}$ and $\Omega$, respectively. Modarres \& Sadi-Nezhad (2001) developed an algorithm for determining preference ratio in TFN cases. An algorithm is also proposed for any continuous cases by Modarres and Sadi-Nezhad (2005). Also to make two fuzzy numbers $A$ and $B$ equivalent by preference ratio they introduced the following definition.

\section{Definition 2:}

a) Two fuzzy numbers $A$ and $B$ are equal by preference ratio criteria if $R(A)=R(B)=0.5$, where $R(A)$ and $R(B)$ are the preference ratio of $A$ and $B$, respectively and the equivalence by preference ratio is shown as $A \stackrel{P R}{\equiv} B$.

b) If $\mathrm{k} A \stackrel{P R}{\equiv} B$, then we say $k$ is the equivalence multiplier of $A$ with respect to $B$.

Note: In the remaining parts of this paper, when we say two fuzzy numbers are equal, we mean they are equal by preference ratio criteria.

Lemma 1. Let $k \neq 0$ be a real number and $A$ and $B$ two fuzzy numbers.

a) If $A$ and $k B$ are equal (by preference ratio criteria), so are $\frac{1}{k} A$ and $B$.

b) If $k_{1}>k_{2}$, then $R\left(k_{1} A\right) \mid \geq R\left(k_{2} A\right)$.

Next section, we propose a new method to obtain an equivalence multiplier to make two fuzzy numbers equivalent. 


\section{Fuzzy AHP by Preference Ratio}

A traditional AHP method is based on the following four main phases,

1- Problem decomposition (hierarchy): This includes three specifications A) Incorporating intuitive understanding apportionment of the whole into parts. B) Hierarchy levels inserted or eliminated as needed. C) Typically it goes from top (global character) to more specific at the bottom.

2- Evaluation or comparative judgment-pairwise comparisons: That is based on the concept of paired compositions as to their importance to a given criterion that occupies the level immediately above the elements being compared (relative or absolute measurements). It also yields a relative scale of priorities which represents the standing with respect to a criterion independent of other criterion, while these relative weights sum to unity.

3- Synthesis of alternatives (ranking) which are resulted from pairwise comparison tables.

4- Consistency analysis: Consistency is necessary but not sufficient for a good decision. It can be captured Consistency Index (CI) and Consistency Ratio (CR) acceptable if it is less than 10\%.

A multiple attribute decision making (MADM) problem with AHP approach, fuzzy data and fuzzy computation may exist in three forms.

i) Fuzzy attributes which can be the result of any fuzzy calculation such as the life of equipment, internal rate of return in projects, economic risk, political risk and etc.

ii) Fuzzy pairwise comparison between qualitative criteria with linguistic terms.

iii) Fuzzy consistency analysis

Modarres \& Sadi-Nezhad (2005) developed a method to normalize input information of attributes They proposed an algorithm to find crisp and normal weights. Therefore, we focus on the second and the third form (ii and iii).

\subsection{Converting Fuzzy Pairwise Comparisons to Crisp Values}

This part deals with pairwise comparison by linguistic terms, which can be explained by trapezoidal fuzzy numbers. This data can be related to DM's idea about comparison between criteria, sub-criteria or comparison between alternatives based on one criterion. By applying algorithm I, the crisp weights are calculated.

\section{Algorithm I}

Let matrix $\widetilde{A}(n . n)$ be a fuzzy reciprocal matrix related to pairwise comparison based on one criterion and $\widetilde{a}_{i j}=1$. Let $\widetilde{a}_{i j}$ be a trapezoidal fuzzy number and represent a linguistic term and related to pairwise comparison between item $i$ and $j$ based on one criterion.

Step1: Compute the summation of each column of the pairwise comparison matrix.

$$
\widetilde{B}_{j}=\sum_{i=1}^{n} \widetilde{a}_{i j} \quad \text { For } j=1 \text { to } n
$$

Step2: Calculate $c_{i j} \in C_{(n \times n)}$ for all $i$ and $j$ such that $\widetilde{a}_{i j} \stackrel{P R}{\equiv} \widetilde{B}_{j} \times c_{i j}$. (For calculation of each crisp $c_{i j}$ we need to apply the algorithm developed by Madaress and Sadi-Nezhad 2005. This algorithm is presented in appendix A) 
Step3: Calculate $D^{T}=\left(D_{1}, \ldots D_{n}\right)$ such that

$$
D_{i}=\sum_{j=1}^{n} c_{i j} \quad \text { For } i=1 \text { to } n
$$

Step4: Calculate the vector of weights, $W^{T}=\left(w_{1}, w_{2}, \ldots w_{n}\right)$ such that

$$
W_{i}=\frac{D_{i}}{\sum_{i=1}^{n} D_{i}} \quad \text { For } i=1 \text { to } n
$$

Example 1: Consider four alternatives which are compared pairwise, based on one criterion and are shown in a trapezoidal fuzzy matrix as follows,

$$
\left[\begin{array}{cccc}
1 & (1,2,2,3) & (1,3,4,5) & (2,3,5,7) \\
(0.333,0.5,0.5,1) & 1 & (2,3,4,6) & (2,3,3,5) \\
(0.2,0.25,0.333,1) & (0.2,0.333,0.333,0.5) & 1 & (0.5,1,1,2) \\
(0.143,0.2,0.333,0.5) & (0.2,0.333,0.333,0.5) & (0.5,1,1,2) & 1
\end{array}\right]
$$

To find crisp weights ( $w_{i}, \quad i=1$ to 4$)$ we use algorithm I.

Step 1:

$\widetilde{B}_{1}=(1.676,1.95,2.166,3.5) \quad \widetilde{B}_{2}=(2.366,3.583,3.667,5) \quad \widetilde{B}_{3}=(4.5,8,10,14) \quad \widetilde{B}_{4}=(5.5,8,10,15)$

Step 2: Calculation of $\mathrm{C}_{4 \times 4}$

$$
C=\left[\begin{array}{cccc}
0.362 & 0.547 & 0.351 & 0.448 \\
0.262 & 0.273 & 0.419 & 0.338 \\
0.228 & 0.088 & 0.108 & 0.121 \\
0.13 & 0.094 & 0.127 & 0.098
\end{array}\right]
$$

Each item is calculated based on the algorithm developed by Modarres \& Sadi-Nezhad 2005.

Step 3:

$$
D_{i}=\sum_{j=1}^{n} c_{i j} \quad D_{1}=1.709 \quad D_{2}=1.2914 \quad D_{3}=0.344 \quad D_{4}=0.4448
$$

Step 4: In this step we calculate the vector of weights

$$
W_{1}=\frac{D_{1}}{D_{1}+D_{2}+D 3+D_{4}}=\frac{1.709}{3.994}=0.4279 \quad W_{2}=0.3233 \quad W_{3}=0.1364 \quad W_{4}=0.1124
$$

As we can observe, the weights are crisp and normal.

\section{Fuzzy Consistency Ratio}

In this section, we introduce an algorithm for calculation and comparison of fuzzy consistency ratio index. This approach is on the basis of equivalence by preference ratio concept. The algorithm is applicable when the crisp weights are determined. 


\section{Algorithm II:}

Step 0: Let $\mathrm{w}_{\mathrm{j}}$ for $j=1, . ., n$ be the crisp weight for $j$ th attribute or alternative.

Step1: Define fuzzy matrix $\widetilde{H}$ such that:

$$
\widetilde{h}_{i j}=w_{j} \cdot \widetilde{a}_{i j}
$$

Step2: Let $\widetilde{s}_{i}$ be the summation of row $i$ in matrix $\widetilde{H}$

$$
\widetilde{s}_{i}=\sum_{j=1}^{n} \widetilde{h}_{i j}
$$

Step 3: Find $\tilde{\lambda}_{i}$ (for $\mathrm{i}=1$ to $\mathrm{n}$ ) such that:

$$
\tilde{\lambda}_{i}=\frac{\widetilde{s}_{i}}{w_{i}} \quad \text { For } i=1 \text { to } n
$$

Step 4: Find CI such that:

$$
C \tilde{I}=\frac{\frac{1}{n} \sum \tilde{\lambda}_{i}-n}{n-1}
$$

( $n$ is the dimension of the matrix)

Step 5: Find fuzzy number $\widetilde{C} R$ such that:

$$
\widetilde{C} R=\frac{\widetilde{C} I}{R C}
$$

(In this formula RC is random consistency)

Step 6: Compare fuzzy number $\widetilde{C} R$ and 0.1 or any ratio that needed with preference ratio and judge.

In step 4 when $\widetilde{a}_{i j}$ is trapezoidal fuzzy number then $\widetilde{h}_{i j}, \widetilde{s}_{i}, \widetilde{\lambda}_{i}, C \widetilde{I}$ and $\widetilde{C} R$ are trapezoidal fuzzy numbers too. In this method we can apply $\sum w_{i} \tilde{\lambda}_{i}$ instead of $\frac{1}{n} \sum \tilde{\lambda}_{i}$ in step 4 . Next example shows the details of the implementation for the second algorithm.

Example 2: To find fuzzy consistency ratio in example 1, apply algorithm II as follows:

Step 0 : The crisp and normal weights are as follows,

$$
W_{1}=0.4279 \quad W_{2}=0.3233 \quad W_{3}=0.1364 \quad W_{4}=0.1124
$$

Step 1 : Using equation (8), the fuzzy matrix $\widetilde{H}$ is calculated as

$$
\widetilde{H}=\left[\begin{array}{cccc}
0.4279 & (0.323,0.646,0.646,0.97) & (0.137,0.41,0.55,0.682) & (0.225,0.337,0.562,0.787) \\
(0.141,0.213,0.213,0.42) & 0.323 & (0.273,0.41,0.55,0.82) & (0.225,0.337,0.337,0.562) \\
(0.086,0.094,0.141,0.428) & (0.065,0.11,0.11,0.162) & 0.1367 & (0.056,0.112,0.112,0.225) \\
(0.06,0.086,0.141,0.213) & (0.065,0.11,0.11,0.162) & (0.068,0.137,0.137,0.273) & 0.112
\end{array}\right]
$$

Step 2: Calculate $\widetilde{s}_{i}$ using Eq. 9

$\widetilde{s}_{1}=(1.113,1.821,2.186,2.867)$

$$
\widetilde{s}_{2}=(0.962,1.283,1.4233,2.133)
$$


$\widetilde{s_{3}}=(0.3437,0.4531,0.5,0.951)$

$$
\widetilde{S}_{4}=(0.3054,0.445,0.5,0.76)
$$

Step 3: Find $\tilde{\lambda}_{i}$ using Eq. 10

$$
\begin{array}{ll}
\tilde{\lambda}_{1}=\frac{\widetilde{s}_{1}}{w_{1}}=(2.6,4.256,5.109,6.7) & \tilde{\lambda}_{2}=\frac{\widetilde{s}_{2}}{w_{2}}=(2.975,3.968,4.402,6.597) \\
\tilde{\lambda}_{3}=\frac{\widetilde{s}_{3}}{w_{3}}=(2.514,3.314,3.658,6.957) & \tilde{\lambda}_{4}=\frac{\widetilde{s}_{4}}{w_{4}}=(2.717,3.959,4.448,6.76)
\end{array}
$$

Step 4: Calculate $\widetilde{C I}$ using Eq. 11

$$
\widetilde{C} I=\frac{\frac{1}{n} \sum \tilde{\lambda}_{i}-n}{n-1}=(-0.433,-0.042,0.135,0.918)
$$

Step 5: $\quad \widetilde{C} R=\frac{\widetilde{C} I}{R C}=\frac{\widetilde{C} I}{0.89}=(-0.486,-0.047,0.152,1.031)$

This is a trapezoidal fuzzy number, see Fig. 1.

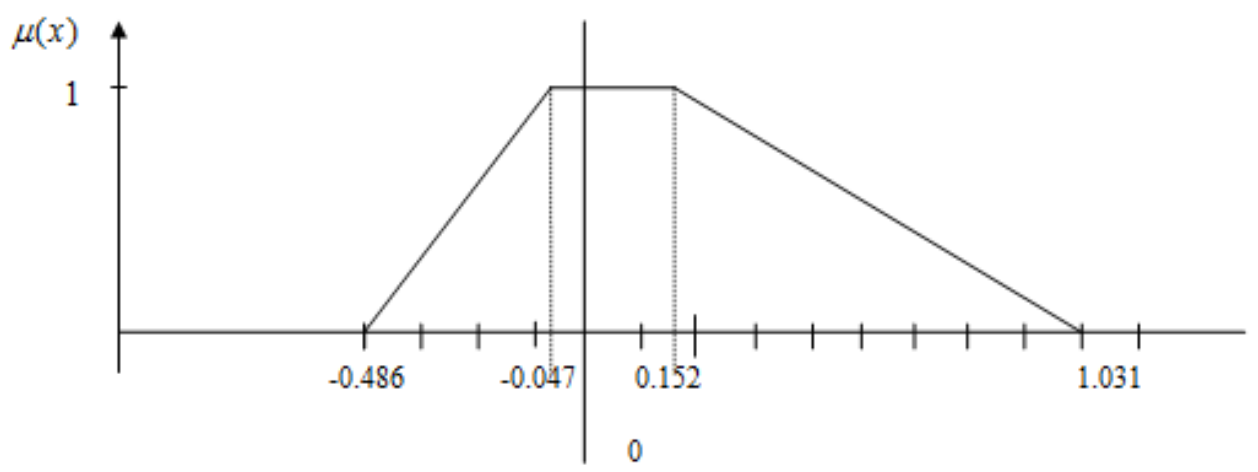

Fig 1 : Fuzzy consistency ratio as a trapezoidal fuzzy number

Step 6: In this step we compare fuzzy number $N_{1}=C \widetilde{R}$ with crisp number

$N_{2}=(0.1,0.1,0.1,0.1)$ by preference ratio.

The result is calculated based on Eq.4 and we have $R(1)=0.36, R(2)=0.64$. Obviously, this is not quite consistent. In other words, $C \widetilde{R}$ is equal to 0.3 by preference ratio which is not a good consistency ratio.

\section{Case study}

It is vital in any educational department to evaluate the offered short courses as well as to prioritize them. To reach this goal, our proposed method is applied in the training center of Industrial Management Institute, an Iranian educational system for training managers. However, in this section rather than presenting the real case, we express the procedure with a much smaller size, for illustration only. In this case, most of the criteria used to compare different alternatives are qualitative. Therefore, linguistic variables seem to be more useful for pair-wise comparisons. Consequently, "fuzzy AHP method by preference ratio" technique is used to make these comparisons more practical. The following steps are taken to prioritize the short courses by applying Fuzzy AHP method.

1- Extracting the criteria and develop appropriate hierarchy. 
2- Fuzzification linguistic variables

3- Performing pair-wise comparison

4- Calculating relative and final weights

The main goal in the hierarchy is "to select the best training short course" in the training center of Industrial Management Institute. Next levels of the hierarchy contain the criteria and the indices for that goal.

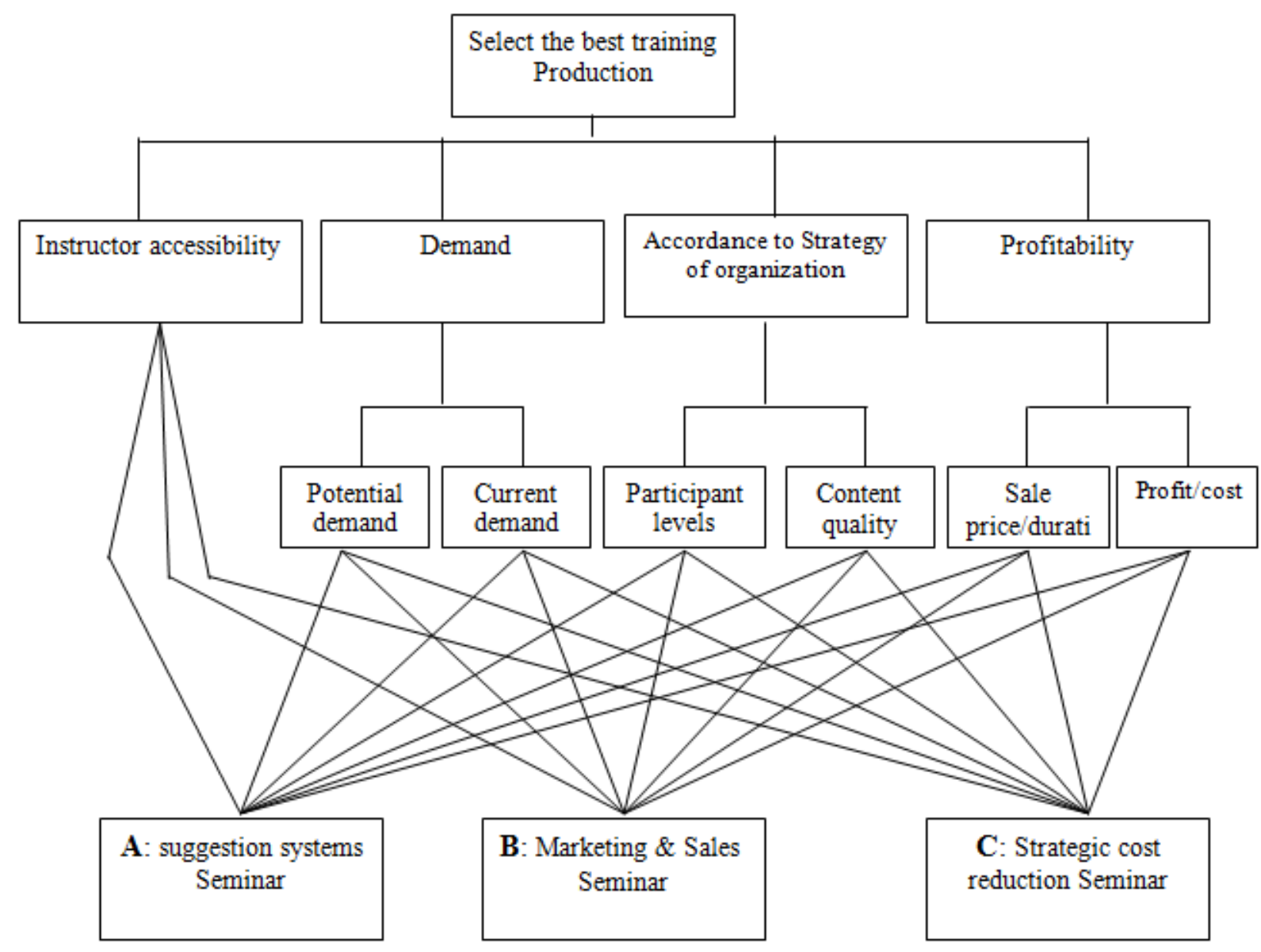

Fig 2: The hierarchy for ranking training seminars in the training center

\subsection{Extracting the criteria and developing the adequate hierarchy for the case:}

First, the list of the needed people who are potential participants is provided.

Those people are:

1- Marketing research group

2- Members of R\&D committee

3- Training managers and head of training center

According to Saaty (1996) "the hierarchy should be large enough to capture your major concerns and small enough to remain sensitive to change in what is important". Fig. 2 demonstrates the details of the hierarchy proposed for the case study of this paper. 


\subsection{Fuzzifying linguistic variables}

To perform pair-wise comparison among all criteria, a questionnaire was designed, based on AHP standard format and Hwang and Chen (1992) scales are used given in Table 1.

Table 1

Qualitative criteria evaluation

\begin{tabular}{lllllll}
\hline $\begin{array}{l}\text { Linguistic } \\
\text { Variable }\end{array}$ & $\begin{array}{l}\text { The same } \\
\text { importance }\end{array}$ & $\begin{array}{l}\text { A little bit } \\
\text { important }\end{array}$ & $\begin{array}{l}\text { Moderate } \\
\text { Important }\end{array}$ & Important & $\begin{array}{l}\text { Very } \\
\text { important }\end{array}$ & Extreme \\
\hline $\begin{array}{l}\text { TFN } \\
(\mathrm{L}, \mathrm{M}, \mathrm{U})\end{array}$ & $(1,1,1)$ & $(1,1,3)$ & $(1,3,5)$ & $(3,5,7)$ & $(5,7,9)$ & $(7,9,9)$ \\
\hline
\end{tabular}

Table 2 shows the details of three short courses in terms of their duration, prices, profits and costs.

\section{Table 2}

The characterization of three different courses

Course characteristics

\begin{tabular}{llllc}
\hline Course & Duration (Day) & Price (Rials) & Profit (Rials) & Cost (Rials) \\
A & 3 & $18,000,000$ & $4,500,000$ & $13,500,000$ \\
B & 2 & $13,000,000$ & $2,600,000$ & $10,400,000$ \\
C & 1 & $8,000,000$ & $1,200,000$ & $6,800,000$ \\
\hline
\end{tabular}

Table 3 demonstrates the details of pairwise comparison in terms of different criteria.

Table 3

Pairwise comparison

\begin{tabular}{llll}
\hline Demand & Potential demand & Current demand & weight \\
\hline Potential demand & TFN $(1,1,1)$ & TFN $(2.1,3.1,5)$ & 0.725 \\
Current demand & TFN $(1 / 5,1 / 3.1,1 / 2.1)$ & TFN $(1,1,1)$ & 0.275 \\
\hline $\begin{array}{l}\text { Accordance to strategy } \\
\text { org }\end{array}$ & Participant level & Content quality & weight \\
\hline Participant level & TFN $(1,1,1)$ & & 0.28 \\
Content quality & TFN $(2,3,5)$ & TFN $(1 / 5,1 / 3,1 / 2)$ & 0.72 \\
\hline Profitability & Seal cost/duration & TFN $(1,1,1)$ & weight \\
\hline Seal cost/duration & 1 & 1 & 0.5 \\
Profit/cost & 1 & 1 & 0.5 \\
\hline
\end{tabular}


Table 4 shows the details of the pairwise comparison between three products of $A, B$ and $C$.

\section{Table 4}

Pairwise comparison

\begin{tabular}{lllll}
\hline Instructor accessibility & Product A & Product B & Product C & weight \\
\hline Product A & 1 & 5 & $5 / 7$ & 0.21 \\
Product B & $1 / 5$ & 1 & $1 / 7$ & 0.06 \\
Product C & $7 / 5$ & 7 & Product C & \\
\hline Current. Demand & Product A & Product B & $(1 / 3,1,1)$ & 0.13 \\
\hline Product A & $(1,1,1)$ & $(1 / 7,1 / 5,1 / 3)$ & $(3,5,7)$ & 0.7 \\
Product B & $(3,5,7)$ & $(1,1,1)$ & $(1,1,1)$ & 0.18 \\
Product C & $(1,1,3)$ & $(1 / 7,1 / 5,1 / 3)$ & Product C & \\
\hline Potential demand & Product A & Product B & $(1,1,3)$ & 0.18 \\
\hline Product A & $(1,1,1)$ & $(1 / 7,1 / 5,1 / 3)$ & $(3,5,7)$ & 0.7 \\
Product B & $(3,5,7)$ & $(1,1,1)$ & Product C & \\
\hline participant level & Product A & Product B & $(1 / 9,1 / 7,1 / 5)$ & 0.1 \\
\hline Product A & $(1,1,1)$ & $(1 / 5,1 / 3,1)$ & $(1 / 7,1 / 5,1 / 3)$ & 0.2 \\
Product B & $(1,3,5)$ & $(1,1,1)$ & $(1,1,1)$ & 0.7 \\
Product C & $(5,7,9)$ & $(3,5,7)$ & Product C & 0.1 \\
\hline content quality & Product A & Product B & $(1 / 9,1 / 7,1 / 5)$ & 0.1 \\
\hline Product A & $(1,1,1)$ & $(1 / 5,1 / 3,1)$ & $(1,1,1)$ & \\
Product B & $(1,3,5)$ & $(3,5,7)$ & & \\
Product C & & & & \\
\hline & & $(5,7,9)$ & & \\
\hline
\end{tabular}

Note that the inconsistency ratios for instructor accessibility, current demand and potential demand, participant level and content quality are calculated as 0.11 , TFN $(-0.362,0.04,0.59)$, TFN $(-0.362$, $0.04,0.59)$, TFN $(-0.372,-0.015,0.72)$ and TFN $(-0.372,-0.015,0.72)$, respectively. The sales price per duration for each product $A, B$ and $C$ are calculated as $0.29,0.32$ and 0.39 , respectively. The profit per cost for each product of $A, B$ and $C$ are also calculated as $0.43,0.33$ and 0.24 , respectively. We have implemented the proposed algorithm of this paper on this case study and the following are the summary of the results,

$$
\lambda 1=(2.91,3.8,4.8) \quad \lambda 2=(3.11,4.42,6.55) \quad \lambda 3=(3.02,4.42,6.46) \quad \lambda 4=(3.28,4.25,7.28)
$$

and the consistency ratio is $C I=(-0.29,0.15,0.76)$ with $R_{1}=0.494$ and $\quad R_{2}=0.506$. 
$R_{1}$ and $R_{2}$ are fuzzy in nature and they must be compared with the consistency value of 0.1 using preference ratio techniques. Since $\left|R_{1}-R_{2}\right|<0.01$ we can conclude that the results are consistent. In the next step, pair-wise comparison of level 2 criteria and pair-wise comparison of alternatives are performed. The sub criteria at all levels 2 and alternatives of hierarchy are compared based on the criteria of level 1. Finally, the priorities of these three short term course are calculated as $\mathrm{W}_{\mathrm{A}}=0.18$, $\mathrm{W}_{\mathrm{B}}=0.29$ and $\mathrm{W}_{\mathrm{C}}=0.55$. As we can observe, the one-day short course has the highest priority while the three-day course program has the least priority and the two-day short course program has the medium priority.

\section{Conclusion}

In this paper, we have developed a new method for fuzzy multiple attribute decision-making in the framework of analytical hierarchy process and the method has been developed by applying the concept of preference ratio. We have also proposed an algorithm for the calculation of fuzzy consistency ratio and the implementation of our proposed method has been demonstrated using a numerical example. The proposed method of this paper has also been applied for a real-world problem of selecting management short courses in a training program of a business school to offer to executive managers.

\section{Reference}

Buckley, J. (1985). Fuzzy Hierarchical Analysis. Fuzzy Sets and Systems, 17(3), 233-247.

Buckley.J, Feuring.T \& Hayashi. Y.(2001). Fuzzy hierarchical analysis revisited. European Journal of Operational Research, 129, 48-64.

Chang, P-L., \& Chen, Y-C.(1994). A Fuzzy multi - criteria decision making method for technology transfer strategy selection in bio technology. Fuzzy Sets and Systems, 64, 131-139.

Chang, D.Y. (1996). Application of the extent analysis method on fuzzy AHP. European Journal of Operational Research,116, $649-655$.

Chen, S. J. \& C. L. Hwang (1992)., Fuzzy Multiple Attribute Decision Making. Springer-Verlag,

Cheng, C.H. (1996). Evaluating Naval tactical Missile Systems by Fuzzy AHP based on the Grade Value of Membership function. European Journal of Operational Research, 96, 343-350.

Cheng, C-H, Yang, K-L \& Hwang, C-L. (1999). Evaluating attack helicopter by AHP based on linguistic variable. European Journal Operational Research, 423 - 435.

Deng, Y.\& Chang, Y-H. (2000). Fuzzy multi criteria analysis for performance evaluation of bus companies. European Journal of Operational Research, 126, 459-473.

Huang, C-C., Chu, P-Y. \& Chiang, Y-H. (2008). A fuzzy AHP application in government-sponsored R\&D project selection. Omega, 36 (6), 1038-1052.

Huang, L-C. \& Wu, R. (2005). Applying fuzzy analytic hierarchy process in the managerial talent assessment model - an empirical study in Taiwan's semiconductor industry. International Journal of Technology Management, 30(1-2), 105-130.

Laarhoven, P.J.M., \& W.Pedrycz. (1985). A Fuzzy Extension of Saaty’s Priority Theory. Fuzzy Sets and Systems, 11(3), $229-241$.

Leung, L.C. \& Cao., D. (2000). On consistency and ranking of alternatives in fuzzy AHP. European Journal of Operational Research, 126, 459-473.

Lin, H-F., Lee, H-S \& Wang, D. W. (2009). Evaluation of factors influencing knowledge sharing based on a fuzzy AHP approach. Journal of Information Science, 35 (1), 25-44.

Mikhailov. L. (2004). A fuzzy approach to driving priorities from interval pair wise comparison judgments. European Journal of Operational Research, 159, 687-704.

Mikhailov. L \& Tsvetinov, P. (2004). Evaluation of services using a fuzzy analytic Hierarchy Process. Applied Soft Computing, 5, 23-33. 
Modarres. M \& Sadi-Nezhad, S. (2001). Ranking fuzzy numbers by preference ratio. Fuzzy Sets and Systems, 118, 429-436.

Modarres, M. \& Sadi-Nezhad, S. (2005). Fuzzy simple additive weighting method by preference ratio. Intelligent Automation and Soft Computing. 11, 235-244.

Saaty, T. L. (1977). A scaling method for priorities in hierarchical structures. Journal of Mathematical Psychology, 15, 234-281.

Saaty,T.L. (2006). Rank from comparisons and from rating in the analytic hierarchy/network. European Journal of Operational Research, 168, 557-570.

Saaty, T. L. \& Vargas, L. (1994) Decision making with analytic hierarchy process. RWS.

Saaty,T. L. (1996). The analytic hierarchy process. RWS.

Şen, C. G \& Çinar, G. (2010). Evaluation and pre-allocation of operators with multiple skills: A combined fuzzy AHP and max-min approach. Expert Systems with Applications, 37, 2043-2053.

Torfi, F., Farahani, R. Z \& Rezapour, S. (2010). Fuzzy AHP to determine the relative weights of evaluation criteria and fuzzy TOPSIS to rank the alternatives. Applied Soft Computing Journal, $10(2), 520-528$.

Yeh ,Deng \& Yo- Hern Chang. (2000). Fuzzy multi criteria analysis for performance evaluation of bus companies. European Journal of Operational Research, 126, 459-473

Zhu, K. J., Jing, Y. \& Chang, D. Y. (1999). A discussion on Extent Analysis Method and applications of Fuzzy AHP. European Journal of Operational Research, 116, 450-456

\section{Appendix A}

\section{Algorithm for making a pair of fuzzy numbers equivalence}

Let $N 1$ and $N 2$ be two non-negative fuzzy numbers. In case $N 1$ and $N 2$ are negative, re-scale the numbers to make them non-negative. First, set $\delta$ as the desired measure of accuracy of the algorithm.

Then, determine $R(N 1)$ and $R(N 2)$.

Step 1. Find $k$ and $k$ from (a) or (b).

Step 2. Calculate $k=(k+k) / 2$.

Step 3. Determine $R(k N 1)$ and $R(N 2)$.

Step 4. If $R(k N 1)<\delta-0.5$, then set $k=(k+k) / 2$. Go to step 2. If $R(k N 1)>\delta+0.5$, then set $k=(k$ $+k) / 2$. Go to step 2. If $|R(N 1)-0.5|<\delta$, stop.

a) If $R\left(N_{1}\right)<R\left(N_{2}\right)$, then $\bar{k}=\max \left\{\frac{L_{2}}{U_{1}}, 1\right\}$ and $\bar{k}=\frac{U_{2}}{L_{1}}$

b) If $R\left(N_{2}\right)<R\left(N_{1}\right)$, then $\underline{k}=\frac{L_{2}}{U_{1}}$, and $\bar{k}=\max \left\{\frac{U_{2}}{L_{1}}, 1\right\}$, 